\section{Vitrectomy for juvenile uveitis: prognostic factors for the long-term functional outcome}

Introduction

Childhood uveitis is a serious and potentially blinding disease, frequently involving both eyes. It is believed that children with uveitis are at greater risk to develop visually significant complications like macular oedema, secondary cataract, or glaucoma. ${ }^{1}$ Corticosteroids are the primary treatment option in noninfectious forms of uveitis. The supplementary treatment with immunomodulatory drugs is advocated if the inflammation is not sufficiently controlled, but also in distinct autoimmune and immunogenetic entities or as a steroid-sparing therapy. $^{2}$

Pars plana vitrectomy (PPV) is nowadays an important treatment option in cases with functional sequelae induced by chronic ocular inflammations (vitreous opacities, cystoid macular oedema (CME), vitreous haemorrhage, retinal detachment, and epiretinal membrane formation) or in cases nonresponsive to immunosuppressive therapy. There is evidence from numerous studies that PPV can lead to an improvement in visual acuity and reduce CME, and uveitic exacerbations. ${ }^{3-16}$ There are only three reports focusing on therapeutic PPV in a paediatric population (in total 43 eyes) with chronic uveitis. ${ }^{17-19}$ All three groups reported a postoperative improvement in visual acuity, a CME reduction, and reduced rate of recurrences with a subsequently achieved reduction of the immunomodulatory therapy after follow-up times of 6 months to 10 years. Furthermore, all findings in juvenile populations are in agreement with the findings in adults with chronic endogenous uveitis after PPV. Therefore, it seems generally accepted that PPV is a valuable therapeutic approach in paediatric uveitis. ${ }^{17-19}$ The follow-up times of these studies, however, were shorter than in our present series.
This work was presented in
part at the symposium 'Eye Surgery/Nitrectomy - Past, Present and Future', 26.-28.2.2004, Bern, Switzerland

\begin{abstract}
Keywords: pars plana vitrectomy; uveitis; children; prognostic factors; cystoid macular oedema clinically significant CME were the most Eye (2006) 20, 184-190. doi:10.1038/sj.eye.6701845; published online 1 April 2005
\end{abstract}


The aim of our retrospective study was to analyse the long-term visual results and the anatomical outcome after PPV in a paediatric population with intermediate uveitis or retinal vasculitis and to identify possible prognostic factors associated with a beneficial final visual outcome.

\section{Patients and methods}

We analysed the clinical records of 23 consecutive paediatric and juvenile patients (29 eyes, 14 right and 15 left) with chronic uveitis who underwent a PPV for significant visual disturbances at the Vitreoretinal Division, Department of Ophthalmology, University Bern between March 1984 and June 2002.

The mean age at the time of surgery was 14.5 years (range, 7.7-19.9 years) with an age distribution as followed: 6-10 years, five patients (21.7\%); 11-15 years, five patients $(21.7 \%)$; $16-20$ years, 13 patients $(56.6 \%)$. In all, 10 patients were females and 13 males. The mean duration of the uveitis was 4.2 years (range, $0.3-11.8$ years). The mean age at onset of the uveitis was 10.4 years (range, 3.8-19.5 years) and the mean follow-up time was 6.3 years (range, $0.2-17.8$ years).

The clinical diagnosis was chronic endogenous intermediate uveitis in 18 (22 eyes) and retinal vasculitis in five patients (including three patients (four eyes) with primary retinal vasculitis and two patients (three eyes) with a secondary retinal vasculitis due to Behçet's disease).

Three eyes had undergone a previous cataract extraction, two with a posterior chamber intraocular lens implantation (IOL) and one was left aphakic. Two eyes had a history of glaucoma treated with topical medications. The primary reasons for PPV were visually relevant vitreous opacities in 25 eyes, vitreous haemorrhage in three eyes, and retinal detachment in one eye.

Patients were examined preoperatively (baseline) to confirm the clinical diagnosis. At each subsequent visit decimal visual acuity (DVA), slit-lamp biomicroscopy, applanation tonometry, and full fundus examination were recorded. All patients had received corticosteroid and/or immunosuppressive therapy before PPV for three or more months.

CME was clinically assessed pre-, intra- and postoperatively. Only a few patients had fluorescein angiography (FA) and therefore CME assessment was based on clinical findings only. To assess the impact of the PPV on CME, we compared the pre- and intraoperative findings with the CME condition at 12 months after surgery.

Cellular infiltration and flare in the vitreous body were graded on a scale from 0 to $4+$ according to the generally accepted International Uveitis Study Group classification, revised by Bloch-Michel and Nussenblatt ${ }^{20}$ and Foster and Vitale. ${ }^{21}$ The number of relapses of chronic uveitis over the total pre- and postoperative observation period was recorded and expressed as number of exacerbations per 12 months' intervals.

Visual acuity was recorded in all patients as DVA and converted into a $\log$ MAR equivalent (log of the minimum angle of resolution; $\log \mathrm{MAR}=-\log$ (decimal acuity)) for statistical purposes. For this, we assumed that hand motion and counting fingers in $60 \mathrm{~cm}$ corresponds approximately to visual acuities of 0.001 and 0.01 , respectively. ${ }^{22}$ For the analysis of the postoperative visual function we evaluated the logMAR visual acuity at the end and the best logMAR visual acuity ever achieved during the follow-up period.

An improvement or loss of more than two DVA steps was regarded as significant.

A standard three-port PPV was performed in all 29 eyes by one of two retinal surgeons (FK, JGG).

Additionally, membrane peeling was accomplished in six eyes, encircling scleral buckle procedures in five eyes, $\mathrm{SF}_{6}$ gas tamponade in seven eyes, transscleral cryocoagulation in eight eyes. A combined lensectomy-vitrectomy approach with posterior chamber IOL implantation was performed in one eye. All patients received a combined topical corticosteroid/ antibiotic preparation during the first 4 weeks after the PPV.

\section{Statistical analysis}

All data are expressed as means \pm standard deviation (SD). All variables showed a skewed distribution (Shapiro-Wilk normality test). The visual acuity course was analysed with the Friedman test and additionally with Dunn's multiple comparison test. For comparisons of categorical data between subgroups of eyes Fisher's two-sided exact test was applied. Comparisons between paired samples were performed using Wilcoxon's rank-sum test. Correlations between variables were assessed by calculating Spearman's rank correlation coefficient.

Multiple regression analysis was performed to identify independent predictive variables (preoperative logMAR acuity, age at surgery, age at uveitis onset, uveitis duration until surgery, posterior vitreous detachment (PVD), CME, gender, cataract, systemic steroids, systemic immunosuppressive medication, grade of vitreous inflammation, vitreous opacities, type of uveitis, and preoperative inflammation recurrences) that influenced the final visual outcome (dependent variable) and their order of importance (Table 1). 
Table 1 Summary of variables used for multiple regression analysis

\begin{tabular}{lcc}
\hline & Mean $\pm S D$ & Range \\
\hline Preoperative logMAR acuity & $0.91 \pm 0.67$ & $0-2.0$ \\
Postoperative logMAR acuity & $0.33 \pm 0.37$ & -0.1 to 1.3 \\
Age at surgery & $14.5 \pm 4.2$ years & $7.7-19.9$ years \\
Age at uveitis onset & $10.4 \pm 4.5$ years & $3.85-19.45$ years \\
Uveitis duration until surgery & $4.2 \pm 3.6$ years & $0.3-11.8$ years \\
Follow-up & $6.3 \pm 4.7$ years & $0.2-17.8$ years \\
Preoperative vitreous inflammation & $1.63 \pm 0.78$ & $0-4.0$ \\
& & $\%$ \\
& $n$ eyes (n total) & 69 \\
Posterior vitreous detachment & $20(29)$ & 34.5 \\
Cystoid macular oedema (preoperative) & $10(29)$ & 58.6 \\
Systemic steroids & $17(29)$ & 13.8 \\
Systemic immunosuppressive Medication & $4(29)$ & 86.2 \\
Vitreous opacities & $25(29)$ & 75.9 \\
Intermediate uveitis & $22(29)$ & 24.1 \\
Retinal vasculitis & $7(29)$ & 62.5 \\
Preoperative uveitis relapses & $15(24)$ & 50 \\
Cataract & $13(26)^{\mathrm{a}}$ & \\
\hline
\end{tabular}

aThree eyes had previous cataract extraction.

Only variables which showed a statistical correlation with the final $\log$ MAR acuity (dependant variable) were chosen as possible independent predictive variables (preoperative logMAR visual acuity, CME, follow-up time, and vitreous opacities). The multiple regression analysis was performed with a forward stepwise inclusion of variables.

For statistical purposes, the variables gender, CME, PVD, cataract, systemic steroids, immunosuppressive medication, vitreous opacities, and the type of uveitis (intermediate uveitis or retinal vasculitis) were introduced as categorical variables (eg, $0=$ male; $1=$ female). Statistical evaluations were conducted using the GraphPad Prism 4 (GraphPad Software, San Diego, USA). A probability of $P \leq 0.05$ was considered significant. All statistical tests were two-sided.

\section{Results}

\section{Visual acuity}

The DVA improved in $25(86.2 \%)$, was stable in two $(6.9 \%)$, and decreased postoperatively in two $(6.9 \%)$ of 29 eyes (Figure 1).

In all, $21(72.4 \%)$ of 29 eyes gained more than two DVA lines during the follow-up period. The number of eyes where the DVA was 0.2 or less reduced from 18 to 8 (Fisher's exact test: $P=0.0167)$, and the number of eyes with a DVA of 0.4 or better increased from 8 to 17 (Fisher's exact test: $P=0.033$ ) after PPV.

The mean logMAR acuity improved postoperatively from $0.91(\mathrm{SD}, 0.67)$ to 0.33 (SD, 0.37). This recovery was

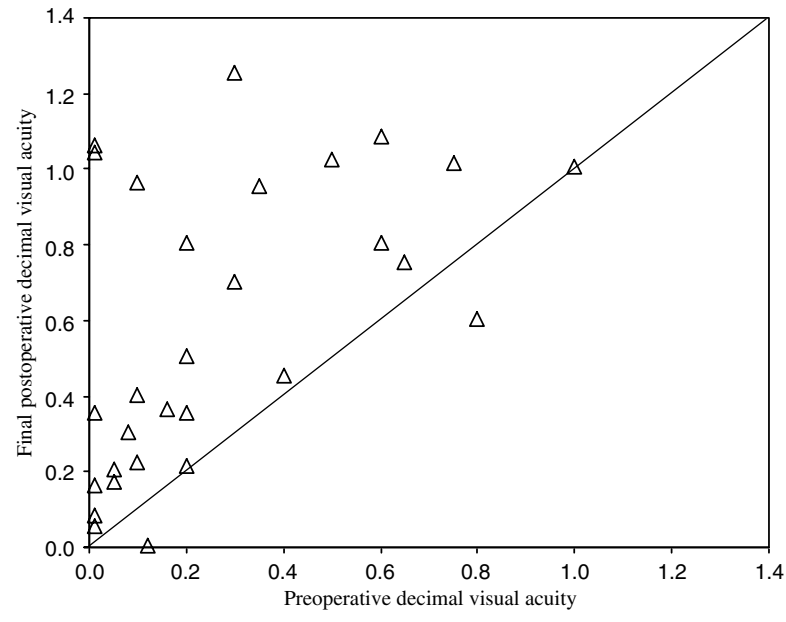

Figure 1 Initial and final postoperative decimal visual acuity following pars plana vitrectomy $(n=29$ eyes $)$.

statistically significant (Friedman test: $P<0.001$ ) and approximately equivalent to a change in DVA from 0.12 (6/50 Snellen acuity) to 0.47 ( $\sim 6 / 13$ Snellen acuity). The best postoperative logMAR acuity was 0.30 (SD, 0.45) corresponding to a DVA of 0.5 (Snellen acuity 6/12) during the follow-up period (Figure 2). There was no visual acuity difference between the uveitis subgroups (intermediate uveitis vs retinal vasculitis).

Between the final $\log$ MAR acuity and the variables preoperative logMAR acuity, CME, cataract, follow-up time, and vitreous opacities a significant correlation was found (preoperative logMAR acuity: $r=0.55, P=0.002$; CME: $r=0.43, P=0.03$; cataract: $r=0.41, P=0.043$, 
follow-up time: $r=-0.48, P=0.01$; vitreous opacities: $r=0.37, P=0.05$ ).

The results of the multiple regression analysis are depicted in Table 2.

Only two of the five chosen independent variables, preoperative logMAR acuity $(P<0.002)$ and CME $(P=0.05)$ were significantly related to the final postoperative $\log$ MAR acuity in a way that a low preoperative $\log$ MAR acuity and the existence of a CME had a negative influence on the final $\log$ MAR acuity. The high multiple correlation coefficient $(R=0.66)$ indicates a strong linear correlation between the observed and the regression model predicted final $\log M A R$ values. About $44 \%$ (coefficient of determination $R^{2}=0.44$ ) of the variation of the final logMAR acuity is explained by the regression model (ANOVA for the regression model, $P<0.002)$.

\section{Cystoid macular oedema}

Clinically significant CME was significantly reduced after PPV from 10 (34.5\%; eight eyes with chronic intermediate uveitis, two eyes with retinal vasculitis) to two eyes $(6.9 \%$; two eyes with chronic intermediate uveitis) at 12 months postoperatively (Fisher's exact test,

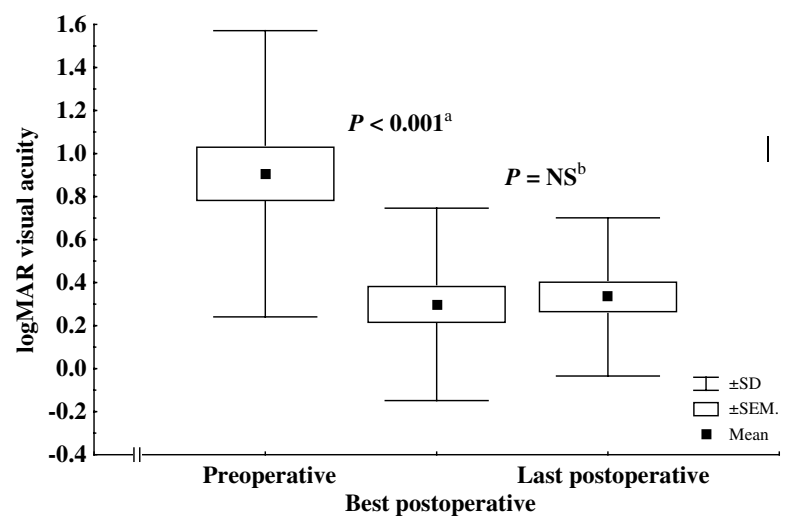

Figure 2 Comparison of the mean $\log$ MAR visual acuity course. Friedman test and Dunn's multiple comparison test; a preoperative $v s$ best postoperative logMAR visual acuity; ${ }^{b}$ best postoperative $v s$ last postoperative logMAR visual acuity $(n=29$ eyes).
$P=0.021)$. The resolution of the CME was, however, not associated with a greater visual improvement. Seven out of eight eyes where the CME regressed in contrast to one of two eyes where it persisted showed an improvement of more than two DVA lines (Fisher's exact test, $P=1.0$ ). One eye without a CME before surgery developed an oedema 21 months after PPV.

\section{Vitreous}

A PVD was observed in $20(69 \%)$ of 29 eyes at the time of surgery. The grade of vitreous inflammatory cell accumulation measured on the last preoperative examination reached an index of 1.63 (SD, 0.78), which decreased to 0.116 weeks after PPV (Wilcoxon signedrank test, $P<0.001)$.

\section{Relapses of chronic uveitis}

There was a significant reduction of chronic uveitis relapses from $15(62.5 \%)$ of 24 eyes before surgery to seven $(29.2 \%)$ eyes after PPV (Fisher's exact test, $P<0.042)$.

Among the eyes with uveitis relapses, a reduction from 11 eyes preoperatively to six eyes after surgery with one to two relapses per 12 months and a reduction from four eyes to one eye with two to three relapses per 12 months was found.

Table 3 Systemic anti-inflammatory and immunosupressive treatment

\begin{tabular}{|c|c|c|}
\hline \multirow[t]{2}{*}{ Treatment } & \multicolumn{2}{|c|}{ Number of eyes } \\
\hline & Preoperative & Postoperative \\
\hline Prednisone $\mathrm{e}^{\mathrm{a}}$ & $17(58.6 \%)$ & $9(31 \%)$ \\
\hline Cyclosporin $^{\mathrm{b}}$ & $1(3.4 \%)$ & $1(3.4 \%)$ \\
\hline Cyclosporin $^{\mathrm{a}}+$ methotrexate $^{\mathrm{c}}$ & $2(3.8 \%)$ & $2(3.8 \%)$ \\
\hline Cyclophosphamid $^{\mathrm{d}}$ & $1(3.4 \%)$ & $1(3.4 \%)$ \\
\hline
\end{tabular}

Table 2 (A) Multiple regression results showing the association between preoperative logMAR acuity and preoperative CME (independent predictor variables) and the final logMAR acuity (dependent variable) and (B) Multiple regression model

\begin{tabular}{lccclcr}
\hline Dependent variable & $\mathrm{R}$ & $\mathrm{R}^{2}$ & ANOVA P-value & Predictor variable & Regression coefficient B & P-value \\
\hline Final logMAR visual acuity & 0.66 & 0.44 & 0.001 & Preoperative logMAR visual acuity & 0.32 & 0.002 \\
& & & & CME & 0.25 & 0.05
\end{tabular}

Final $\log$ MAR visual acuity $=0.32 \times$ preoperative $\log$ MAR visual acuity $+0.25 \times \mathrm{CME}-0.03$ 


\section{Immunosuppressive treatment}

The need for systemic steroid treatment was not significantly reduced from $17(58.6 \%)$ of the eyes preoperatively to nine (31\%) eyes postoperatively (Fisher's exact test, $P=0.07)$. Four (13.8\%) eyes required immunomodulatory drugs (cyclosporine, methotrexate, or cylophosphamide) before and after PPV (Table 3).

\section{Cataract}

A cataract extraction was performed in the later postoperative follow-up period in five of 13 eyes with pre-existing lens opacities (one eye underwent a combined lensectomy-vitrectomy) and in three eyes without a pre-existing cataract.

In total, 11 eyes (three eyes pre-PPV, one eye combined lensectomy-vitrectomy, seven eyes post-PPV) received a cataract extraction with IOL implantation in eight eyes. Three eyes were left aphakic.

The functional influence of cataract formation on final $\log$ MAR visual acuity is emphasised by a significant correlation ( $r=0.41, P=0.043)$. In the multiple regression model the factors cataract and vitreous opacities are represented by the preoperative $\log$ MAR acuity.

\section{Postoperative complications}

The most severe and deleterious complication was the development of a painful phthisis bulbi in one eye with a subsequent enucleation and a preoperative chronic ocular hypotonia and a DVA of 0.12 .

Two eyes of two patients had to be reoperated twice because of a retinal detachment with proliferative vitreoretinopathy (PVR). One of them had a pre-existing retinal detachment. The final DVAs in these cases were 0.08 and 0.05 , respectively.

\section{Discussion}

According to the published and our own results, PPV has a beneficial effect on the course and complications of chronic uveitis in paediatric and juvenile patients with respect to the anatomical and visual outcome. ${ }^{17-19}$

A stable gain of more than $2 \log$ MAR acuity lines was observed in more than two-thirds of the eyes and maintained over a follow-up of more than 6 years, which is clearly beyond previous studies. The logMAR visual acuity in our study group improved significantly from 0.91 (DVA 0.12) to 0.3 (DVA 0.5) after PPV. These results compare well with other reports focusing on PPV in paediatric populations, where an increase of DVA from $0.19-0.3$ preoperatively to $0.53-0.72$ after surgery was achieved. ${ }^{17-19}$
Furthermore, we found an improvement of the DVA in 86.2\% of the eyes after PPV, which compares favourably with a recent meta-analysis of 58 scientific papers (1934 eyes) about PPV in adult uveitis patients, where a visual acuity improvement in $83 \%$ of the eyes was found. ${ }^{23}$

Multiple regression analysis revealed that only two of the tested independent predictor variables, namely preoperative logMAR acuity and clinically present CME were significantly associated with the final postoperative $\log$ MAR acuity in our population. The factors vitreous opacities and cataract are indirectly contained in this regression model by negatively influencing the preoperative logMAR visual acuity.

Clinically detectable CME decreased in $80 \%$ of our cases after PPV, which compares well with previously reported findings, showing CME regression rates of 87.5 and $100 \%$ in paediatric populations. ${ }^{17,18}$ Several studies with adult patients showed a greater variation of the CME regression rate (32.4-82.4\%). ${ }^{4-6,9,10,12}$ The abovementioned meta-analysis showed a regression rate of 47.4\% after PPV. ${ }^{23}$ This fact may suggest a better inflammatory CME resolution rate in paediatric patients than in adults after PPV.

One of the limitations of our retrospective approach was the solely clinical evaluation of the CME in most of the cases and the lack of documented evidence of CME regression by angiography. A subclinical form of $\mathrm{CME}$ may have persisted after a gross regression of the oedema, which was sufficient enough to prevent a visual acuity improvement. Furthermore, post-CME macular changes (atrophic changes of the retinal pigment epithelium and the retina after long-standing CME) may limit VA improvement despite clinical CME regression or resolution. Multiple regression implies that a pre-existing CME is a very important prognostic factor and has a negative impact to the final $\log M A R$ visual acuity. This finding is in line with previously published reports in adults. ${ }^{4,6}$ With the present regression model, $44 \%$ (coefficient of determination, $R^{2}=0.44$ ) of the variation of the final $\log$ MAR acuity is thereby explained. The remaining part of the variation must be explained by other unknown individual patient factors. Interestingly, neither the preoperative uveitis duration nor the age at uveitis onset or surgery seemed to influence the final visual acuity in our study population. Furthermore, the CME regression rate was maybe influenced by the continuation of immunomodulatory treatment in the postoperative period.

In the present study, we found a significantly reduced relapse rate of chronic uveitis in the postoperative period compared to the preoperative time and a trend towards a reduced systemic corticosteroid demand. These findings compare well with several studies pertaining to vitrectomy in paediatric and adult uveitis which have 
found a reduction in the recurrence rate and the severity of inflammation with a subsequent reduction of the immunosuppressive need in the postoperative course. $^{4,5,9-12,17-19}$ A possible explanation for these results could be the restoration of an immunological homeostasis by the mechanical removal of the vitreous body with captured autoreactive T cells and proinflammatory cytokines during PPV. ${ }^{24,25}$ The replacement of the vitreous with aqueous humor and its numerous immunomodulatory factors after PPV may have an additional regulatory effect. ${ }^{26}$ In contrast, the need for systemic steroids and immunomodulatory treatment showed no significant difference before and after PPV.

The most severe complication we had encountered was an eye with chronic intermediate uveitis and a hypotonia preoperatively, which progressed to a phthisis bulbi within 12 months postoperatively. A subsequent enucleation had to be performed due to a painful eye with light perception only. This complication has first been described by Klöti. ${ }^{15}$ In 2 eyes, RD with PVR (preexisting in one of them) required reoperations.

A postoperative cataract extraction was required in five of 13 eyes with preoperative lens opacities (one eye underwent a combined lensectomy-vitrectomy). Only three eyes with preoperatively clear lenses developed a cataract after PPV. These findings are in agreement with the reported frequency of cataract development in adult series. ${ }^{8,9,27}$ In the present study, the increase of the $\log$ MAR acuity after PPV is maybe partly explained by cataract extractions in the postoperative period.

PPV has a beneficial effect on the course and the complications of chronic uveitis in paediatric and juvenile patients. The most important effects are an improvement of $\log$ MAR visual acuity, a reduction of CME and a reduced relapse rate of chronic uveitis after PPV. Preoperative logMAR visual acuity and clinically significant $\mathrm{CME}$ were the most accurate predictors for the functional outcome in a way that a low preoperative logMAR visual acuity and a pre-existing CME had a negative impact on final logMAR visual acuity.

We propose PPV as a surgical treatment option in all cases where immunosuppressive medication fails to improve visual function and to resolve inflammatory activity.

Further studies with more patients and a controlled design are mandatory to investigate the effect of PPV in paediatric uveitis patients; however, the paucity of cases hampering makes the realisation of a prospective study difficult.

\section{Acknowledgements}

We would like to thank Dr Robert MacLaren, Institute of Ophthalmology, University College London for critical reading of this manuscript.

\section{References}

1 Cunningham Jr ET. Uveitis in children. Ocul Immunol Inflamm 2000; 8: 251-261.

2 Holland GN, Stiehm ER. Special considerations in the evaluation and management of uveitis in children. Am J Ophthalmol 2003; 135: 867-878.

3 Radetzky S, Walter P, Fauser S, Koizumi K, Kirchhof B, Joussen AM. Visual outcome of patients with macular edema after pars plana vitrectomy and indocyanine greenassisted peeling of the internal limiting membrane. Graefes Arch Clin Exp Ophthalmol 2004; 242: 273-278.

4 Scott RA, Haynes RJ, Orr GM, Cooling RJ, Pavesio CE, Charteris DG. Vitreous surgery in the management of chronic endogenous posterior uveitis. Eye 2003; 17: 221-227.

5 Stavrou P, Baltatzis S, Letko E, Samson CM, Christen W, Foster CS. Pars plana vitrectomy in patients with intermediate uveitis. Ocul Immunol Inflamm 2001; 9: 141-151.

6 Wiechens B, Nolle B, Reichelt JA. Pars-plana vitrectomy in cystoid macular edema associated with intermediate uveitis. Graefes Arch Clin Exp Ophthalmol 2001; 239: 474-481.

7 Bovey EH, Herbort CP. Vitrectomy in the management of uveitis. Ocul Immunol Inflamm 2000; 8: 285-291.

8 Thumann G, Bartz-Schmidt KU, Esser P, Walter P, Kirchhof $\mathrm{B}$, Krott $\mathrm{R}$ et al. Vitrektomie in der Behandlung von Augen mit komplizierter Uveitis. Klin Monatsbl Augenheilkd 1997; 211: $241-244$

9 Verbraeken H. Therapeutic pars plana vitrectomy for chronic uveitis: a retrospective study of the long-term results. Graefes Arch Clin Exp Ophthalmol 1996; 234: 288-293.

10 Heiligenhaus A, Bornfeld N, Foerster MH, Wessing A. Long-term results of pars plana vitrectomy in the management of complicated uveitis. Br J Ophthalmol 1994; 78: 549-554.

11 Messerli J, Koerner F, Ruggli J. Chronische Uveitiden: Verlauf nach Vitrektomie. Klin Monatsbl Augenheilkd 1992; 200: 378-381.

12 Eckardt C, Bacskulin A. Vitrectomy in intermediate uveitis. Dev Ophthalmol 1992; 23: 232-238.

13 Mieler WF, Aaberg TM. Vitreous surgery in the management of peripheral uveitis. Dev Ophthalmol 1992; 23: 239-250.

14 Heimann K, Schmanke L, Brunner R, Amerian B. Pars plana vitrectomy in the treatment of chronic uveitis. Dev Ophthalmol 1992; 23: 196-203.

15 Kloeti R. Pars plana-Vitrektomie bei chronischer Uveitis. Klin Monatsbl Augenheilkd 1988; 192: 425-429.

16 Diamond JG, Kaplan HJ. Lensectomy and vitrectomy for complicated cataract secondary to uveitis. Arch Ophthalmol 1978; 96: 1798-1804

17 Kroll P, Romstock F, Grenzebach UH et al. Frühvitrektomie bei endogener juveniler Uveitis media - Eine Langzeitstudie. Klin Monatsbl Augenheilkd 1995; 206: 246-249.

18 Bacskulin A, Eckardt C. Ergebnisse der pars planaVitrektomie. Ophthalmologe 1993; 90: 434-439.

19 Ulbig M, Kampik A. Pars plana-Vitrektomie bei chronischer Uveitis des Kindes. Klin Monatsbl Augenheilkd 1989; 194: 10-12.

20 Bloch-Michel E, Nussenblatt RB. International Uveitis Study Group: recommendations for the evaluation of intraocular inflammatory disease. Am J Ophthalmol 1987; 103: 234-235. 
21 Foster CS, Vitale TA. Diagnosis of uveitis. In: Foster CS, Vitale TA (eds) Diagnosis and Treatment of Uveitis. W.B. Saunders Company: Philadelphia, PA, 2002, p 92.

22 Holladay JT. Proper method for calculating average visual acuity. J Refract Surg 1997; 13: 388-391.

23 Becker MD, Harsch N, Zierhut M, Davis JL, Holz FG. Therapeutische Vitrektomie bei Uveitis. Aktueller Stand der Empfehlungen. Ophthalmologe 2003; 100: 787-795.

24 Dick AD, Carter DA. Cytokines and immunopathogenesis of intraocular posterior segment inflammation. Ocul Immunol Inflamm 2003; 11: 17-28.
25 Muhaya M, Calder VL, Towler HM, Jolly G, McLauchlan M, Lightman S. Characterization of phenotype and cytokine profiles of $\mathrm{T}$ cell lines derived from vitreous humour in ocular inflammation in man. Clin Exp Immunol 1999; 116: 410-414.

26 Nishida T, Taylor AW. Specific aqueous humor factors induce activation of regulatory T cells. Invest Ophthalmol Vis Sci 1999; 40: 2268-2274.

27 Dev S, Mieler WF, Pulido JS, Mittra RA. Visual outcomes after pars plana vitrectomy for epiretinal membranes associated with pars planitis. Ophthalmology 1999; 106: 10861090. 